\title{
Seqüência de Fluxo de Gás Fresco para Início da Anestesia com Baixo Fluxo: Aplicação Clínica do Estudo Teórico de Mapleson *
}

\author{
Fresh-Gas Flow Sequence at the Start of Low-Flow Anesthesia: \\ Clinical Application of Mapleson's Theoretical Study \\ Marisa Miziara Jreige Borges ${ }^{1}$, Renato Ângelo Saraiva, TSA $^{2}$
}

\begin{abstract}
RESUMO
Borges MMJ, Saraiva RA - Seqüência de Fluxo de Gás Fresco para Início da Anestesia com Baixo Fluxo: Aplicação Clínica do Estudo Teórico de Mapleson
\end{abstract}

\begin{abstract}
Justificativa e Objetivos - Em estudo teórico, Mapleson utilizando um modelo farmacocinético multicompartimental, com um homem padrão de 40 anos e $70 \mathrm{~kg}$, demonstrou que, com fluxo de gás fresco (FGF) inicial igual à ventilação pulmonar total, sendo depois reduzido até $1 \mathrm{~L}^{\mathrm{min}} \mathrm{m}^{-1} \mathrm{e}$ concentração (Fracional) administrada do anestésico $\left(F_{\text {adm }}\right)$ igual a 3 CAM, a fracional expirada final, também expressa como alveolar $\left(F_{E}=F_{A}\right)$, pode atingir $1 \mathrm{CAM}$ em poucos minutos, de acordo com a solubilidade do agente inalado. O objetivo do presente trabalho foi realizar a aplicação clínica deste modelo teórico.
\end{abstract}

Método - Foram incluídos neste estudo 28 pacientes de ambos os sexos, com idade entre 18 e 55 anos, submetidos à anestesia geral, divididos aleatoriamente em 4 grupos de 7 pacientes de acordo com anestésico utilizado (halotano, isoflurano, sevoflurano e desflurano). A indução foi venosa com propofol, fentanil e vecurônio e a manutenção com o agente inalatório diluído em oxigênio, sob ventilação pulmonar mecânica. Os parâmetros foram os seguintes, de acordo com o agente utilizado: Grupo do halotano: FGF inicial de 5 L. $\mathrm{min}^{-1}$ até 4 minutos, seguido por 2,5 L. $\mathrm{min}^{-1}$ até 10 minutos e 1,5 L. min $^{-1}$ até 20 minutos, $F_{\text {adm }}$ igual a 3 CAM durante os 20 minutos iniciais da anestesia. Grupo do isoflurano: O FGF inicial foi de $5 \mathrm{~L} \cdot \mathrm{min}^{-1}$ por 1,5 minuto, seguido por 1,5 L. $\mathrm{min}^{-1}$ até 7 minutos e 1 L. min $^{-1}$ até 20 minutos. A $F_{\text {adm }}$ foi de 3 CAM até 7 minutos e 2,5 CAM até o vigésimo minuto. Grupo do sevoflurano: O FGF inicial foi de $5{\mathrm{~L} . \mathrm{min}^{-1}}^{-1}$ por 1 minuto e 1 L. in $^{-1}$ até o vigésimo minuto e a $F_{\text {adm }}$ de 3 CAM por 1 minuto, depois 2,5 CAM até 7 minutos e 1,8 CAM até 20 minutos. Grupo do desflurano: O FGF inicial foi de 3,5 L. $\mathrm{min}^{-1}$ por 1 minuto e 1 L. in $^{-1}$ até completar os 20 minutos e a $F_{\text {adm }}$ de 3 CAM por 1 minuto, seguido de 1,5 CAM até 10 minutos e 1,2 CAM até 20 minutos. Além da monitorização rotineira das variáveis fisiológicas (cardiovasculares e respiratórias) foram medidas $F I$, e FE' (FA) dos agentes utilizados.

\footnotetext{
* Recebido do (Received from) Departamento de Anestesiologia da Rede SARAH de Hospitais, Brasilia, DF
}

1. Anestesiologista do Hospital SARAH - Brasília-DF

2. Coordenador de Anestesiologia da Rede SARAH de Hospitais

Apresentado (Submitted) em 23 de maio de 2001

Aceito (Accepted) para publicação em 09 de outubro de 2001

Correspondência para (Mail to):

Dr. Renato Ângelo Saraiva

SMHS Quadra 501 - Conjunto A

70335-901 Brasilia, DF

(C) Sociedade Brasileira de Anestesiologia, 2002
Resultados - Grupo do halotano: a FA atingiu 1,15 CAM em 2 minutos e variou de 1,21 a 1,47 CAM até 20 minutos. Grupo do isoflurano: a FA foi de 1,03 CAM em 1 minuto, variando de 1,11 a 1,21 CAM até 20 minutos. Grupo do sevoflurano: a FA de 1,53 CAM foi atingida em 1 minuto, variando de 1,10 a 1,34 CAM até 20 minutos restantes. Grupo do desflurano: a FA foi de 0.94 CAM em 1 minuto, variando de 1,07 até 1,14 CAM até o vigésimo minuto.

Conclusões - Os resultados obtidos comprovam a aplicabilidade clínica do modelo teórico de Mapleson. Deste modo, conseguiu-se um rápido aumento da FA do agente inalatório que atingiu 1 CAM, em 1 a 2 minutos, mantendo-se neste valor com pequenas oscilações e baixo consumo de anestésico.

UNITERMOS - ANESTÉSICOS, Volátil: desflurano, halotano, isoflurano, sevoflurano; TÉCNICAS ANESTÉSICAS, Inalatória: baixo fluxo

\section{SUMMARY}

Borges MMJ, Saraiva RA - Fresh-Gas Flow Sequence at the Start of Low-Flow Anesthesia: Clinical Application of Mapleson's Theoretical Study

Background and Objectives - In a theoretical study, Mapleson using a multicompartmental pharmacokinetic model in a standard 40-year old and $70 \mathrm{~kg}$ man, has shown that with a fresh gas flow (FGF) initially equal to total pulmonary minute ventilation and then decreased to $1 \mathrm{~L}$. $\mathrm{min}^{-1}$, and with fractional anesthetic administration $\left(F_{a d m}\right)$ set to $3 M A C$, the end fractional expired also expressed as alveolar $\left(F_{E},=F_{A}\right)$ may reach 1 MAC in few minutes, according to the solubility of the inhaled agent. The purpose of this study was to clinically apply.

Methods - Twenty-eight patients of both genders, aged 18 to 55 years, scheduled to undergo general anesthesia, were randomly divided in four groups of seven patients each according to the anesthetic drug to be used (halothane, isoflurane, sevoflurane and desflurane). Anesthesia was induced with intravenous propofol, fentanyl and vecuronium, and maintained with the inhalational agent diluted in oxygen under mechanical ventilation. Gas parameters were set, according to the agent as follows: Halothane group: initial FGF of 5 L. min ${ }^{-1}$ up to the $4^{\text {th }}$ minute, followed by $2.5 \mathrm{~L}$. $\mathrm{min}^{-1}$ up to the $10^{\text {th }}$ minute and 1.5 L. min $^{-1}$ up to the $20^{\text {th }}$ minute; $F_{\text {adm }}$ was 3 MAC during the first 20 minutes of anesthesia. Isoflurane group: initial FGF of 5 L. in $^{-1}$ for 1.5 minute, followed by $1.5 \mathrm{~L}$. $\mathrm{min}^{-1}$ up to the $7^{\text {th }}$ minute and 1 L. min $^{-1}$ up to the $20^{\text {th }}$ minute. $F_{\text {adm }}$ was 3 MAC up to the $7^{\text {th }}$ minute and 2.5 MAC up to the $20^{\text {th }}$ minute. Sevoflurane group: initial FGF of 5 L. min $^{-1}$ for 1 minute and 1 L. in $^{-1}$ up to the $20^{\text {th }}$ minute. $F_{a d m}$ was 3 MAC for 1 minute, 2.5 MAC up to 7 minutes and 1.8 MAC up to the $20^{\text {th }}$ minute. Desflurane group: initial FGF of 3,5 L. min ${ }^{-1}$ for 1 minute and 1 L. min $^{-1}$ up to the $20^{\text {th }}$ minute. $F_{\text {adm }}$ was 3 MAC for 1 minute followed by 1.5 MAC up to the $10^{\text {th }}$ minute and 1.2 MAC up to the $20^{\text {th }}$ minute. In addition to routine monitoring of physiological (cardiovascular and respiratory) variables, FI and FE', (FA) of the inhaled agents were measured. 
Results - Halothane group: FA reached 1.15 MAC in 2 minutes and varied from 1.21 to $1.47 \mathrm{MAC}$ until the $20^{\text {th }}$ minute. Isoflurane group: FA reached 1.03 MAC in 1 minute and varied from 1.11 to $1.21 \mathrm{MAC}$ until the $20^{\text {th }}$ minute. Sevoflurane group: FA reached 1.53 MAC in 1 minute and varied from 1.10 to 1.34 MAC until the $20^{\text {th }}$ minute. Desflurane group: FA reached 0,94 MAC in 1 minute and varied from 1.07 to 1.14 MAC until the $20^{\text {th }}$ minute.

Conclusions - Results obtained confirm the clinical feasibility of Mapleson's theoretical model. This way, a fast FA increase of the inhaled agent was achieved, which reached 1 MAC in 1 to 2 minutes and was maintained within this value with minor variations and low anesthetic consumption.

KEY WORDS - ANESTHETICS, Volatile: desflurane, halothane, isoflurane, sevoflurane; ANESTHETIC TECHNIQUES, Inhalational: low-flow

\section{INTRODUÇÃO}

anestesia inalatória com baixo fluxo de gases foi iniciada por John Snow no final do século XIX com intenção de reduzir o consumo e evitar a poluição dos anestésicos, conseguindo diminuir bastante o odor do clorofórmio e do éter nas salas de cirurgias ${ }^{1}$. Ele criou um aparelho experimental de circuito fechado, no qual o paciente respirava oxigênio enquanto o dióxido de carbono exalado era absorvido por hidróxido de potássio. O próprio Snow fez os primeiros testes. Em seguida, Ralph Watters, nos anos vinte, usou o sistema de "vaivém" com fluxos reduzidos ${ }^{2}$ para baixar os custos e elevar a margem de segurança com a administração do ciclopropano, um excelente anestésico gasoso, mas de alto custo, inflamável e explosivo, mesmo em baixas concentrações ${ }^{3}$. Posteriormente este método foi reintroduzido, por Harry Lowe, com fluxos baixíssimos, chamados fluxos basais, no método de anestesia quantitativa ${ }^{4}$.

Abase da administração de fármacosé a dose. Em anestesia inalatória a dose anestésica é medida por frações ou múltiplos da Concentração Alveolar Mínima (CAM), podendo ser 1 CAM o "alvo" inicial para obtenção do estado de anestesia. No método quantitativo, o cálculo da dose em volume de vapor utiliza os valores da CAM, solubilidade do agente, ventilação alveolar e débito cardíaco do paciente. Depois este volume de vapor é transformado em volume de líquido a ser administrado.

Este método, mesmo sendo muito eficiente, é pouco utilizado porque envolve vários cálculos. Então, compreende-se que o anestesiologista, em sua prática clínica, precisa de um método simples que possa utilizar com o equipamento disponível, com segurança e sem o risco de cometer erros de cálculos. Deste modo, fazendo apenas variações de fluxo e concentrações administradas no vaporizador do agente, de acordo com o tempo, seria possível utilizar um fluxo de gás fresco suficientemente baixo para atender suas preocupações quanto ao consumo e poluição ambiental da sala de cirurgia.

Foi demonstrada por Mapleson ${ }^{5}$, a simulação dos primeiros 20 minutos de anestesia, que, com um fluxo de gás fresco inicialmente igual à ventilação total e com uma concentração ou fracional administrada do anestésico $\left(F_{\text {adm }}\right)$ de 3 CAM, a fracional expirada final $\left(F_{E^{\prime}}\right)$ se aproxima de 1 CAM em $1 \mathrm{mi}-$ nuto com desflurano e sevoflurano; em 1,5 minutos com isoflurano; em 2,5 minutos com enflurano e em 4 minutos com halotano.

O objetivo deste trabalho foi aplicar clinicamente o estudo teórico de Mapleson, que propõe um rápido aumento da fracional expirada final (alveolar) do agente inalatório para que o valor de 1 CAM seja logo atingido e se mantenha constante, com um gasto mínimo de anestésico.

\section{MÉTODO}

Após aprovação pela Comissão de Ética, participaram do estudo 28 pacientes de ambos os sexos, com idade variando de 18 a 55 anos, estado físico ASA I e II, programados para procedimentos cirúrgicos sob anestesia geral com ventilação pulmonar mecânica.

Os pacientes foram divididos, aleatoriamente, em quatro grupos de sete pacientes, de acordo com o agente utilizado: halotano; isoflurano; sevoflurano e desflurano.

Foi administrado como medicação pré-anestésica midazolam (15 mg) por via oral. Os pacientes foram monitorizados com cardioscópio, PA não invasiva, oxímetro de pulso, temperatura e capnometria. As fracionais inspirada e expirada final (alveolar) dos anestésicos foram medidas pelo analisador de gases incorporado ao aparelho de anestesia Ohmeda. Após venóclise, foi realizada pré-oxigenação do paciente e do sistema de ventilação durante 3 minutos com $\mathrm{O}_{2}$ a $100 \%$. Em seguida procedeu-se à indução anestésica com os seguintes agentes venosos: propofol (2,5 a $\left.3 \mathrm{mg} \cdot \mathrm{kg}^{-1}\right)$; fentanil $\left(1 \mu \mathrm{g} \cdot \mathrm{kg}^{-1}\right)$ e vecurônio $\left(0,1 \mathrm{mg} \cdot \mathrm{kg}^{-1}\right)$ e a manutenção anestésica com agente inalatório único diluído em oxigênio. Aseqüência de fluxo de gás fresco e a fracional administrada dos anestésicos, de acordo com o modelo de Mapleson, está na tabela I. Iniciou-se o estudo com os vaporizadores abertos para administrar uma concentração equivalente a 3 CAM do agente anestésico. Foi utilizado também um gráfico de conversão da concentração dos vários anestésicos em CAM, de acordo com a idade ${ }^{6}$ e corrigidos para a altitude de 1000 metros e pressão atmosférica de $700 \mathrm{mmHg}$ (Figura 1). No grupo do halotano com um FGF de 5 L. min $^{-1}$ e uma $F_{\text {adm }}$ equivalente a 3 CAM até o quarto minuto. A fracional inspirada aumentou no primeiro minuto e se manteve pouco abaixo de 3 CAM. Após 4 minutos, o fluxo foi reduzido para 2,5 L. $\mathrm{min}^{-1} \mathrm{e}$ após 10 minutos para 1,5 L. $\mathrm{min}^{-1}$ permanecendo até 20 minutos. $\mathrm{AF}_{\text {adm }}$ foi mantida em $3 \mathrm{CAM}$. No grupo do isoflurano foi utilizado um fluxo de gás fresco inicial de $5 \mathrm{~L}$. $\mathrm{min}^{-1}$ por 1,5 minuto e, a seguir reduzido para $1,5 \mathrm{~L} \cdot \mathrm{min}^{-1}$ até o sétimo minuto e $1 \mathrm{~L}$. $\mathrm{min}^{-1}$ até o vigésimo minuto. $A F_{\text {adm }}$ foi de 3 CAM até o sétimo minuto e 2,5 até o vigésimo minuto. No grupo do sevoflurano o fluxo de gás fresco inicial foi de $5 \mathrm{~L}$. $\mathrm{min}^{-1}$ e a $F_{\text {adm }}$ de 3 CAM no primeiro minuto. Em seguida, o fluxo foi reduzido para $1 \mathrm{~L}$. $\mathrm{min}^{-1}$ até completar 20 minutos e a $F_{\text {adm }}$ reduzida para 2,5 CAM até o sétimo minuto e para 1,8 CAM até o final. No grupo do desflurano, o fluxo de gás fresco inicial foi de $3,5 \mathrm{~L}$. $\mathrm{min}^{-1}$ e a $\mathrm{F}_{\mathrm{adm}}$ de $3 \mathrm{CAM}$ no primeiro minuto. Posteriormente o fluxo foi reduzido para $1 \mathrm{~L} \cdot \mathrm{min}^{-1}$ até 20 minutos, e a 
$F_{\text {adm }}$ reduzida para 1,5 CAM até 10 minutos e 1,2 CAM até o final.

Tabela I - Seqüência de Fluxo de Gás Fresco e Fracional dos Anestésicos em Múltiplos da CAM Utilizados por Mapleson

\begin{tabular}{lccc}
\hline & Tempo $(\mathrm{min})$ & Fluxo $\left({\left.\mathrm{L} . \mathrm{min}^{-1}\right)}^{-}\right.$ & $\begin{array}{c}\text { Pressão Parcial } \\
\text { (Unidades da CAM) }\end{array}$ \\
\hline Halotano & $0-04$ & 5,0 & 3,0 \\
& $04-10$ & 2,5 & 3,0 \\
Isoflurano & $>10$ & 1,5 & 3,0 \\
& $0-1,5$ & 5,0 & 3,0 \\
& $1,5-07$ & 1,5 & 3,0 \\
Sevoflurano & $>07$ & 1,0 & 2,5 \\
& $0-01$ & 5,0 & 3,0 \\
& $01-07$ & 1,0 & 2,5 \\
Desflurano & $>07$ & 1,0 & 1,8 \\
& $01-01$ & 3,5 & 3,0 \\
& $>10$ & 1,0 & 1,5 \\
& & 1,0 & 1,2 \\
\hline
\end{tabular}

Os pacientes foram mantidos em ventilação pulmonar mecânica com freqüência respiratória $(\mathrm{Fr})$ de 10 ciclos por minuto (c.p.m.) e volume corrente (Vt) de $10 \mathrm{ml}^{\mathrm{kg}} \mathrm{kg}^{-1}$ (100 $\left.\mathrm{ml} \cdot \mathrm{kg}^{-1} \cdot \mathrm{min}^{-1}\right)$.

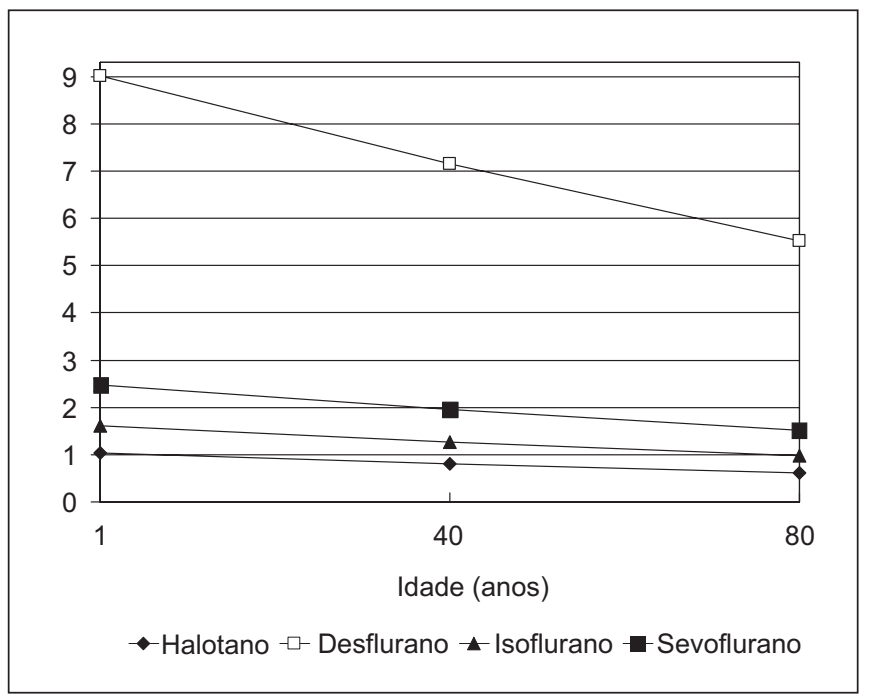

Figura 1 - Representa a Conversão de Pressão Parcial dos Anestésicos em Concentração Alveolar Mínima (CAM), Relacionado com a Idade, corrigidos para altitude de 1000 m e pressão barométrica de $700 \mathrm{mmHg}(101 \mathrm{kPa})$
Os dados foram analisados durante os primeiros 20 minutos de anestesia e o volume líquido de anestésico gasto neste períod o foi medido para todos os anestésicos utilizados, com exceção do desflurano, por impossibilidade técnica (anestésico extremamente volátil, vaporizador e frasco com válvulas). Para o desflurano o volume foi calculado pela fórmula:

$$
\mathrm{V}=\frac{\text { Concentração fluxo tempo }}{\frac{D 22,4(273 t)}{P M 273}}
$$

(Concentração em \%/100, fluxo em $\mathrm{ml} / \mathrm{min}$, tempo em minutos; $\mathrm{V}=$ volume, $\mathrm{D}=$ densidade; $\mathrm{PM}=$ peso molecular; $\mathrm{e}$ $\mathrm{t}=$ temperatura, $\left.\mathrm{em}^{\circ} \mathrm{C}\right)^{4}$.

A análise estatística foi realizada pela análise de variância por meio do teste $\mathrm{F}$, considerando-se um nível de significância $\alpha=0,05(5 \%)$.

\section{RESULTADOS}

Os pacientes não apresentaram diferenças estatísticas significativas entre os grupos, quanto ao peso e a idade (Tabela II). Os dados do consumo de anestésicos foram obtidos por medidas do volume em 20 minutos (Tabela III). E comparando-se aos relatos da literatura pode ser considerado pequeno ${ }^{7}$.

Tabela III - Volume de Anestésico Consumido (ml) em 20 Minutos (Média $\pm \mathrm{DP}$ )

\begin{tabular}{lc}
\hline Grupo & Volume Consumido \\
\hline Halotano & $8,16 \pm 3,42$ \\
Isoflurano & $11,31 \pm 5,08$ \\
Sevoflurano & $9,63 \pm 5,81$ \\
Desflurano * & $11,89 \pm 0,65$ \\
\hline
\end{tabular}

*Volume calculado

Quanto aos parâmetros hemodinâmicos, a pressão arterial média (Figura 2) variou de 98 a $63 \mathrm{mmHg}$. Com uso de halotano, houve um caso que no nono minuto, apresentou uma queda pressórica de 22,56\% (PA-69/35 mmHg), sendo necessário administrar efedrina $10 \mathrm{mg}$, com melhora do quadro (PA-83/41 mmHg). A freqüência cardíaca (Figura 3) variou de 98 a 68 bpm. Foi observado um caso com sevoflurano em que a redução foi de $25,39 \%$ no $17^{\circ}$ minuto, mas sem significância clínica. Em relação aos parâmetros ventilatórios, a fracional de $\mathrm{CO}_{2}$ expirada final (Figura 4) variou de 35 a 30 $\mathrm{mmHg}$, também sem relevância clínica.

Tabela II - Idade e Peso (Média \pm DP)

\begin{tabular}{lccc}
\hline Grupos & Halotano & Isoflurano & Sevoflurano \\
\hline Idade (anos) & $31,14 \pm 11,91$ & $29,57 \pm 8,64$ & $30,86 \pm 12,86$ \\
Peso $(\mathrm{kg})$ & $62,14 \pm 17,32$ & $65,71 \pm 9,18$ & $62 \pm 7,64$ \\
\hline
\end{tabular}




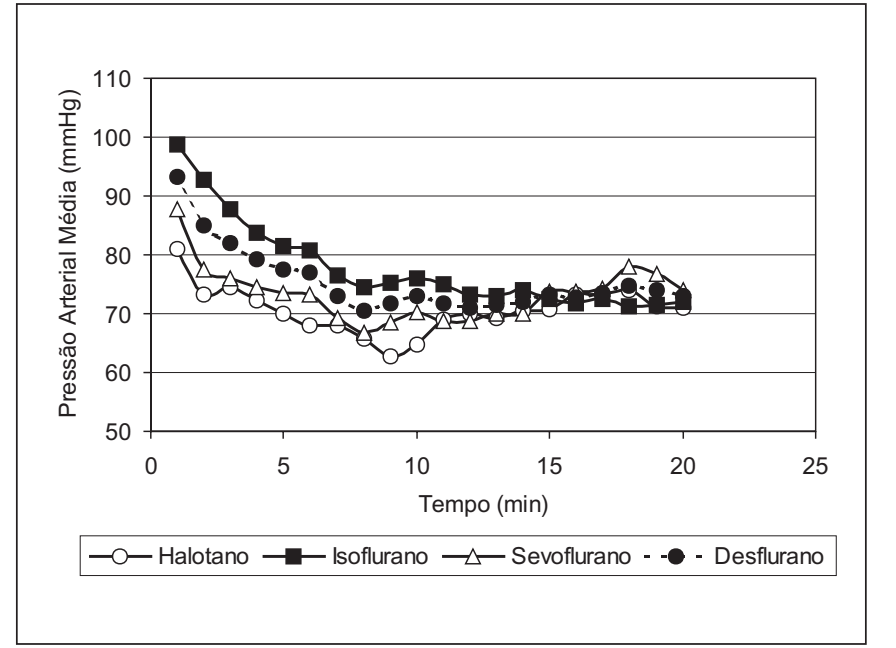

Figura 2 - Pressão Arterial Média Durante os 20 Minutos Iniciais

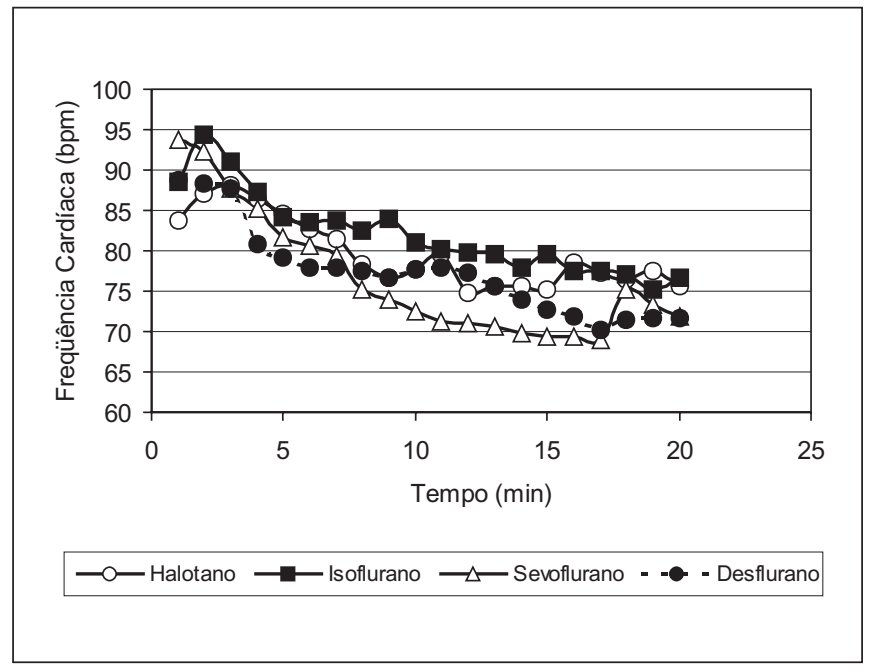

Figura 3 - Freqüência Cardíaca Durante os 20 Minutos Iniciais

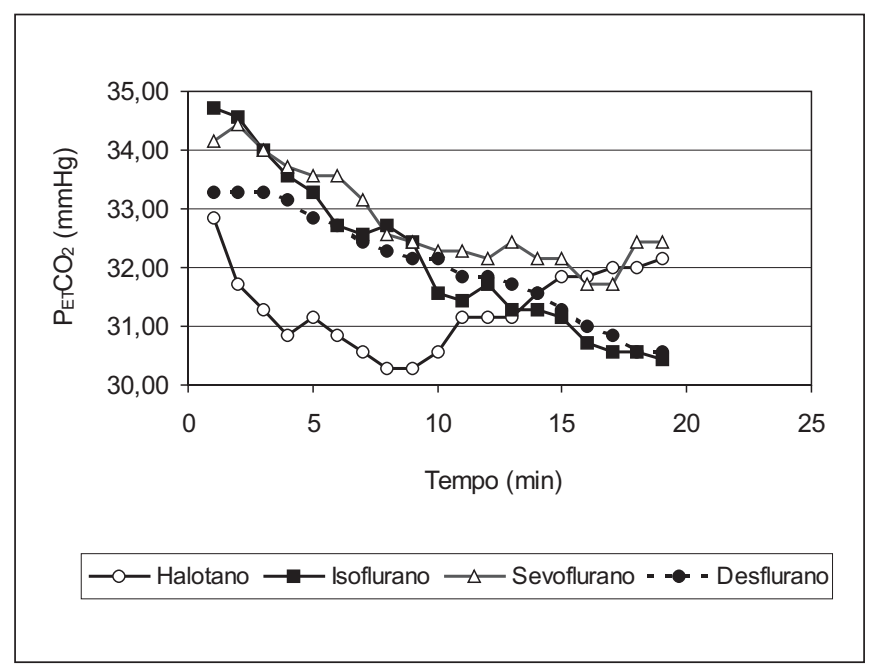

Figura 4 - Fração Expirada de $\mathrm{CO}_{2}$ Durante os 20 Minutos Iniciais
No grupo do halotano, a relação $F_{A} / C A M$ igual a 1, ou seja, 1 CAM foi atingida em 2 minutos, variando de 1,21 a 1,47 CAM durante os 20 minutos iniciais, o que pode ser admitido como valores dentro da normalidade para uma anestesia clinicamente estável, considerando que Dose Anestésica ${ }_{95}\left(\mathrm{DA}_{95}\right)$ é cerca de 1,3 $\mathrm{CAM}^{8}$ (Figura 5).

No grupo do isoflurano, a relação $F_{A} / C A M$ igual a 1 foi atingida com 1 minuto, e variou de 1,11 a 1,21 CAM durante os 20 minutos (Figura 6).

No grupo do sevoflurano, observou-se, no primeiro minuto, relação $F_{A} / C A M$ de 1,53, e $F_{A}$ variando de 1,10 a 1,34 CAM até o vigésimo minuto (Figura 7).

No grupo do desflurano, observou-se uma relação $F_{A} / C A M$ igual a 0,94 com 1 minuto e uma $F_{A}$ que variou de 1,07 a 1,14 CAM (Figura 8) durante os 20 minutos.

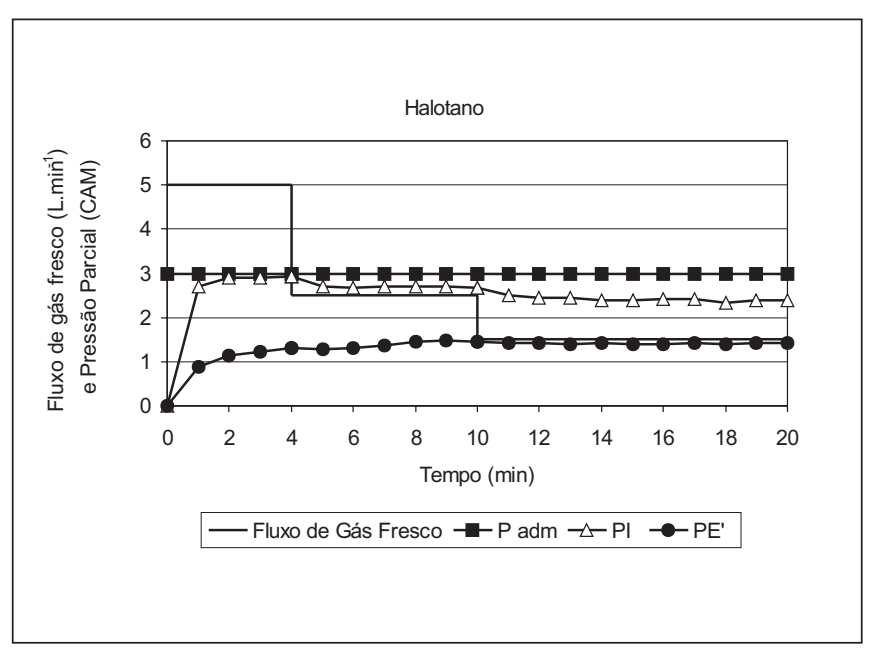

Figura 5 - Resultados Obtidos com a Administração de Halotano

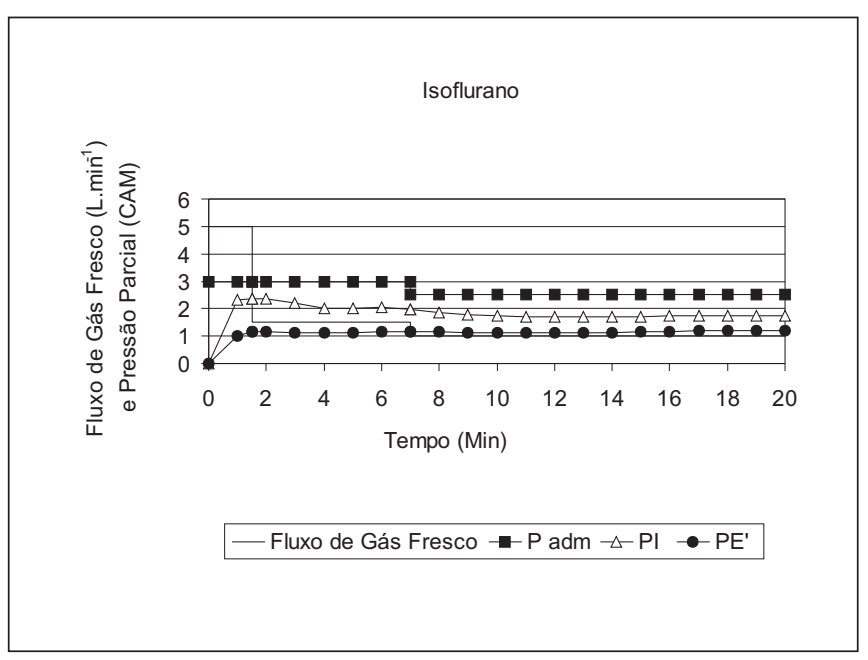

Figura 6 - Resultados Obtidos com a Administração de Isoflurano 


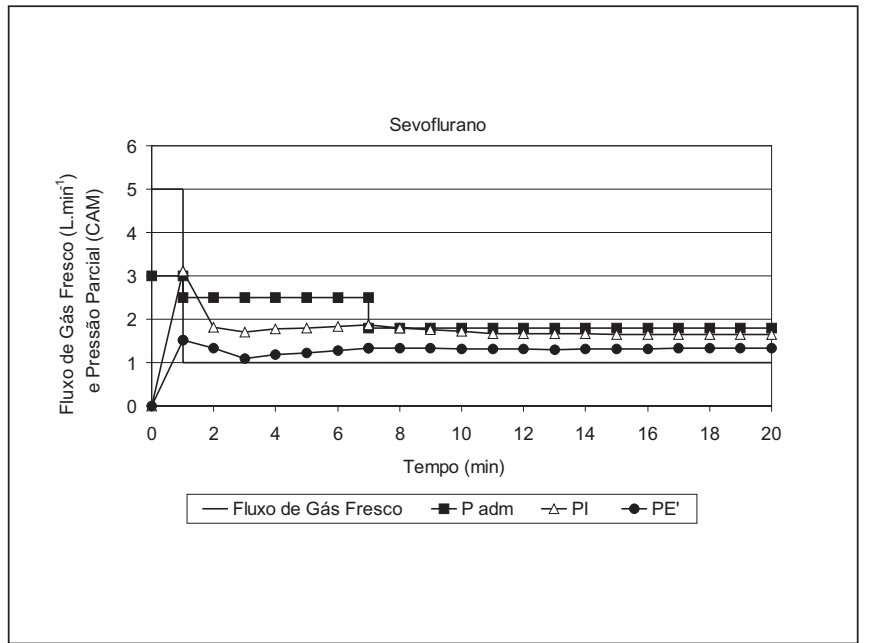

Figura 7 - Resultados Obtidos com a Administração de Sevoflurano

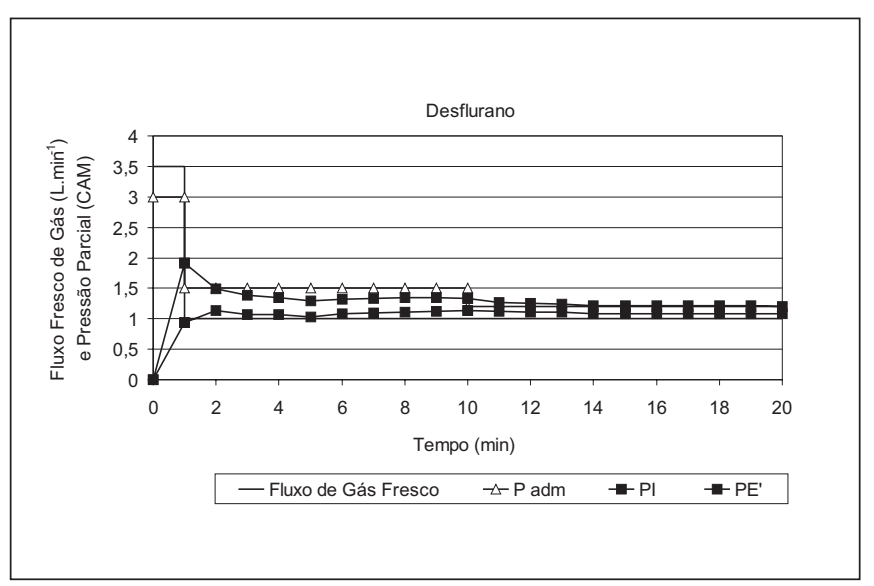

Figura 8 - Resultados Obtidos com Administração de Desflurano

\section{DISCUSSÃO}

De acordo com o estudo teórico de Mapleson, avaliou-se como o fluxo de gás fresco e a concentração (fracional) dos anestésicos poderiam ser melhor utilizados durante os primeiros minutos de anestesia, a fim de se obter sua rápida indução com gasto mínimo de anestésico volátil ${ }^{5}$. Esta rápida indução requer que se inicie com alto fluxo de gás fresco (FGF) e elevada fracional de administração dos anestésicos $\left(F_{a d m}\right)$, para que o processo de equilíbrio cinético entre esta fracional e a fracional inspirada $\left(F_{1}\right)$ se realize rapidamente e, em seguida, através de ventilação alveolar eficiente, a fracional alveolar $\left(F_{A}\right)$ se aproxime da $F_{1}$.

Este modelo teórico é capaz de simular a captação (absorção) e distribuição dos anestésicos voláteis através de processamento de dados obtidos pelas variáveis fisiológicas: ventilação, espaço morto, débito cardíaco e perfusão sangüínea dos órgãos, tomando como base um homem padrão de 40 anos e $70 \mathrm{~kg}$ de peso corporal ${ }^{9}$. Os valores dos volumes dos componentes do aparelho de anestesia e da solubilida- de dos agentes, expressos pelos coeficientes de partição sangue/gás e tecidos/sangue de cada órgão, foram obtidos da literatura ${ }^{10,11}$.

No modelo, o sistema respiratório é tricompartimental, constituído do aparelho de anestesia (inclusive o espaço morto mecânico), o espaço morto anatômico e alvéolo pulmonar. Em seguida, a circulação e os tecidos são multicompartimentais e estão dispostos em série, de acordo com a perfusão sangüínea: cérebro, vísceras, músculos e gorduras. Estes estão ligados à perfusão sangüínea arterial que trás o anestésico do alvéolo para os órgãos, e a perfusão sangüínea venosa, que leva de volta o anestésico para o alvéolo.

A simulação é feita imaginando o anestésico sendo levado em forma de vapor, do vaporizador para o sistema respiratório pelo fluxo de gás fresco e deste ao alvéolo pulmonar pela ventilação alveolar, sendo daí retirado pelo sangue e distribuído para os órgãos de acordo com a perfusão sangüínea e a solubilidade. Neste trajeto, o anestésico sofre o efeito da diluição no aparelho de anestesia e também no alvéolo. A ventilação alveolar é a principal determinante do equilíbrio cinético entre a fracional alveolar e a fracional inspirada. Neste modelo, é prevista a captação de anestésico do alvéolo pelo sangue, que a seguir faz a distribuição para todo o corpo. Sendo a perfusão sangüínea cerebral muito elevada, o equilíbrio cérebro-alveolar logo ocorre. Desta forma, a concentração de anestésico alveolar, ou seja, a fracional expirada final expressa a fracional cerebral.

No início da administração de anestésico inalatório há uma grande diferença entre a fracional administrada, que sai imediatamente do vaporizador, e a fracional inspirada, que está ao nível da boca do paciente após passar pelo sistema de inalação do aparelho de anestesia, bem como entre esta e a fracional alveolar que obviamente está ainda bem abaixo do valor da CAM do agente que está sendo utilizado. Estas diferenças são explicadas pela ausência do anestésico no sistema de inalação do aparelho de anestesia e no ar alveolar neste momento da anestesia.

A obtenção de uma $F_{A}$ igual ou próxima à CAM é a primeira meta a ser atingida na administração de anestésico inalatório. Para isto deve ser feita uma oferta de anestésico bastante elevada para o sistema de inalação através do fluxo de gás fresco e deste para o alvéolo pulmonar pela ventilação alveolar. Considerando este fundamento farmacocinético, o modelo teórico de Mapleson prevê a obtenção da CAM logo nos primeiros minutos, dependendo da solubilidade do agente. Por esta razão, o fluxo de gás fresco é elevado, igual a ventilação pulmonar total e a $F_{\text {adm }}$ igual a 3 CAM. Procurou-se também obter uma ventilação alveolar dentro dos padrões de normalidade, para manter o paciente em normocapnia ou leve hipocapnia e levar ao alvéolo o volume de anestésico necessário para que a CAM seja logo atingida.

Usando-se os valores de FGF e $F_{\text {adm }}$ nos tempos previstos no método e descritos nos resultados foram obtidas as relações $F_{A} / C A M$ que tiveram variações (picos) de 0,94 a 1,5 com média de 1,2. Estes resultados se aproximaram muito do estudo de Mapleson, cujo alvo era uma $F_{A} / C A M$ de 1 a ser obtida nos primeiros minutos, mantendo-se constante a seguir.

Revista Brasileira de Anestesiologia Vol. 52, № 2, Março - Abril, 2002 
O halotano, agente mais solúvel, atingiu a CAM em 2 minutos. Os outros anestésicos (isoflurano, sevoflurano e desflurano) atingiram a CAM em 1 minuto. Sendo estes menos solúveis, o equilíbrio entre a pressão parcial alveolar e a pressão parcial inspirada se processa mais rapidamente.

A diferença entre estes resultados e os previstos no estudo teórico de Mapleson deve-se certamente ao fato de que nossa amostra de pacientes não foi padronizada (homens de 40 anos e $70 \mathrm{~kg}$ ). Foram pessoas comuns, que encontramos em nossa atividade clínica diária. Mesmo assim, deve ser salientado que as diferenças entre os resultados obtidos foram pequenas sem significância estatística.

O consumo de anestésico nos primeiros minutos de anestesia foi elevado devido ao alto fluxo de gás fresco utilizado. A partir do momento em que o fluxo foi reduzido, obviamente o consumo passou a ser baixo. Apesar deste maior volume utilizado no início da anestesia, a média do volume gasto de anestésico nos 20 minutos de avaliação foi pequeno.

É importante esclarecer que para utilizar este método com eficiência e segurança, é recomendável ter disponível um monitor de gases anestésicos que possa medir continuamente as fracionais inspiradas e expiradas finais destes agentes, além do equipamento de anestesia completo, contendo a monitorização padrão com oximetria e capnometria. Atualmente esta aparelhagem deve fazer parte do uso rotineiro do anestesiologista, sempre que possível.

Os resultados obtidos comprovam a aplicabilidade do modelo teórico de Mapleson. Desta maneira, conseguiu-se um rápido aumento da fracional expirada final (alveolar) do agente inalatório, que atingiu 1 CAM logo nos minutos iniciais e depois se manteve constante, podendo o consumo de anestésicos ser considerado entre os menores descritos.

\section{Fresh-Gas Flow Sequence at the Start of Low-Flow Anesthesia: Clinical Applica- tion of Mapleson's Theoretical Study}

Marisa Miziara Jreige Borges, M.D., Renato Ângelo Saraiva, TSA, M.D.

\section{INTRODUCTION}

Low gas flow inhalational anesthesia was introduced by John Snow in the end of $19^{\text {th }}$ Century aiming at decreasing consumption and preventing anesthetic pollution, being able to significantly decrease chloroform and ether odor in operating rooms ${ }^{1}$. He created an experimental close circuit device in which patients would breath oxygen while exhaled $\mathrm{CO}_{2}$ was absorbed by potassium hydroxide. Snow himself performed the first tests. Ralph Watters, during the 20 s, used a "to-and-fro" system with low flows ${ }^{2}$ to reduce costs and increase cyclopropane's safety margin, which is an excellent through high-cost gaseous anesthetic, flammable and explosive even in low concentrations ${ }^{3}$. This technique was later reintroduced by Harry Lowe with very low flows, called basal flows, in a quantitative anesthesia method ${ }^{4}$.

Drug administration is based on dosing. In inhalational anesthesia, doses are measured by fractions or multiples of Minimum Alveolar Concentration (MAC) where 1 MAC might be the initial "target" for inducing anesthesia. In the quantitative method, the dose expressed in vapor volume is calculated from MAC values, agent's solubility, alveolar ventilation and cardiac output. This volume is then converted into volume of anesthetic liquid to be administered.

Although very efficient, this method is seldom used for involving several calculations. This is why anesthesiologists in clinical practice need a simple method to be safely used with the available equipment and with no risk of calculation mistakes. This way, simply adjusting flow and administered concentration in the vaporizer, according to time, it would be possible to use a fresh gas flow low enough to meet the concerns of consumption and surgery room environmental pollution.

Mapleson ${ }^{5}$ has shown in a 20 -minute anesthesia simulation that, with an initial fresh gas flow equal to total minute ventilation and with an anesthetic concentration or fractional $\left(F_{\text {adm }}\right)$ of $3 \mathrm{MAC}$, end expired fractional (FE') is close to $1 \mathrm{MAC}$ in 1 minute with desflurane and sevoflurane, 1.5 minute with isoflurane, 2.5 minutes with enflurane and 4 minutes with halothane.

The purpose of this study was to clinically apply Mapleson's theoretical study, which proposes a fast end expired fractional (alveolar) increase of the inhalational agent to reach 1 $\mathrm{MAC}$, and to maintain it constant there after, with minimum anesthetic consumption.

\section{METHODS}

After the Ethical Committee approval, 28 patients of both genders, aged 18 to 55 years, physical status ASA I and II, scheduled for surgical procedures under general anesthesia with mechanical ventilation were included in this study. Patients were randomly distributed in four groups of seven patients, according to the anesthetic agent used: halothane, isoflurane, sevoflurane and desflurane.

Patients were premedicated with oral midazolam $(15 \mathrm{mg})$. Monitoring consisted of cardioscope, non-invasive BP, pulse oximetry, temperature and capnometry. Anesthetics inspired and end expired (alveolar) fractionals were measured by a gas analyzer built in an Ohmeda monitor. After venoclysis, patients and ventilation system were pre-oxygenated for 3 minutes with $100 \% \mathrm{O}_{2}$. Anesthesia was then induced with propofol (2.5 to $\left.3 \mathrm{mg} \cdot \mathrm{kg}^{-1}\right)$, fentanyl $\left(1 \mu \mathrm{g} . \mathrm{kg}^{-1}\right)$ and vecuronium $\left(0.1 \mathrm{mg} \cdot \mathrm{kg}^{-1}\right)$, and maintained with a single inhalational agent diluted in oxygen.

Anesthetics fresh gas flow and agents fractional sequences according to Mapleson's model, are shown in table I. The study was started with open vaporizers for the administration of concentration equivalent to 3 MAC of the anesthetic agents. A chart was used to convert anesthetics concentra- 
Table I - Fresh Gas Flow Sequence and Anesthetics Fractional in MAC Multiples Used by Mapleson

\begin{tabular}{lccc}
\hline & $\begin{array}{c}\text { Time } \\
(\mathrm{min})\end{array}$ & $\begin{array}{c}\text { Flow } \\
\left(\text { L.min }^{-1}\right)\end{array}$ & $\begin{array}{c}\text { Partial Pressure } \\
(\text { MAC Units })\end{array}$ \\
\hline Halothane & $0-04$ & 5.0 & 3.0 \\
& $04-10$ & 2.5 & 3.0 \\
Isoflurane & $>10$ & 1.5 & 3.0 \\
& $0-1.5$ & 5.0 & 3.0 \\
& $1.5-07$ & 1.5 & 3.0 \\
Sevoflurane & $>07$ & 1.0 & 2.5 \\
& $0-01$ & 5.0 & 3.0 \\
Desflurane & $01-07$ & 1.0 & 2.5 \\
& $>07$ & 1.0 & 1.8 \\
& $01-01$ & 3.5 & 3.0 \\
& $>10$ & 1.0 & 1.5 \\
\hline
\end{tabular}

tions into MAC, according to age ${ }^{6}$. It was adjusted for an altitude of 1000 meters and an atmospheric pressure of 700 $\mathrm{mmHg}$ (Figure 1). In the halothane group, with an FGF of 5 L. min $^{-1}$ and $F_{\text {adm }}$ equivalent to 3 MAC up to 4 minutes, inspired fractional was increased in the first minute and was maintained slightly below $3 \mathrm{MAC}$. After the $4^{\text {th }}$ minute, flow was decreased to $2.5 \mathrm{~L}$. $\mathrm{min}^{-1}$ and after the $10^{\text {th }}$ minute to $1.5 \mathrm{~L} \cdot \mathrm{min}^{-1}$ until the $20^{\text {th }}$ minute. $\mathrm{F}_{\mathrm{adm}}$ was maintained in $3 \mathrm{MAC}$. The isoflurane group received an initial fresh gas flow of $5 \mathrm{~L} \cdot \mathrm{min}^{-1}$

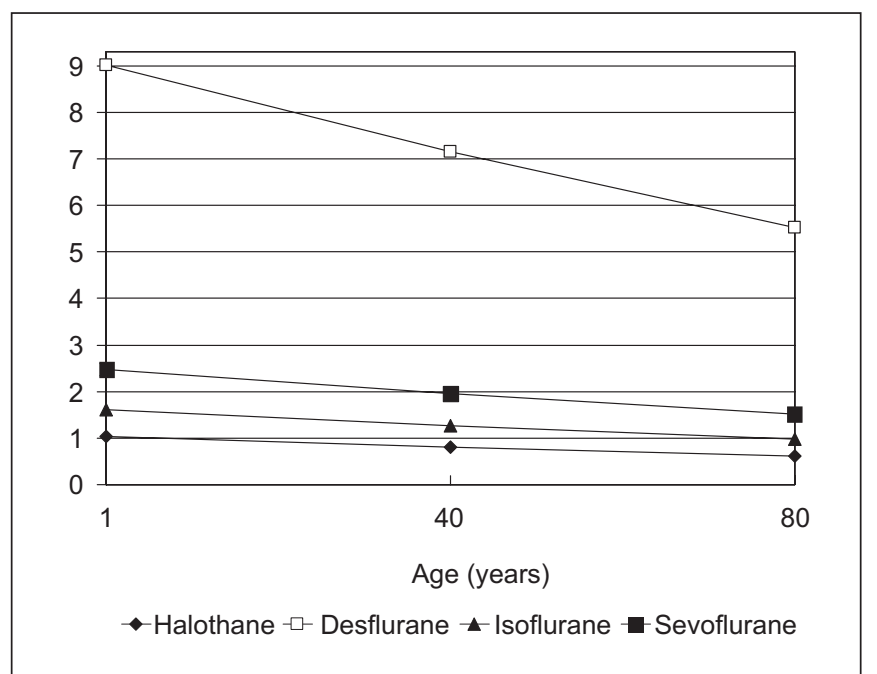

Figure 1 - Anesthetics Partial Pressure Translation into Minimum Alveolar Concentration (MAC), Related to Age, Adjusted for an altitude of $1000 \mathrm{~m}$ and atmospheric pressure of $700 \mathrm{mmHg}$ $(101 \mathrm{kPa})$ for 1.5 minutes which was decreased to $1.5 \mathrm{~L} . \mathrm{min}^{-1}$ until the $7^{\text {th }}$ minute and to $1 \mathrm{~L} \cdot \mathrm{min}^{-1}$ until the $20^{\text {th }}$ minute. $F_{\text {adm }}$ was 3 MAC for 7 minutes and $2.5 \mathrm{MAC}$ until the $20^{\text {th }}$ minute. The sevoflurane group received an initial fresh gas flow of 5 L. min $^{-1}$ and $F_{\text {adm }}$ was of 3 MAC in the first minute. Then, the flow was reduced to $1 \mathrm{~L} \cdot \mathrm{min}^{-1}$ until the $20^{\text {th }}$ minute and $F_{\text {adm }}$ was decreased to $2.5 \mathrm{MAC}$ until the $7^{\text {th }}$ minute and to $1.8 \mathrm{MAC}$ until the end. Initial fresh gas flow for the desflurane group was 3.5 L. min $^{-1}$ and $F_{\text {adm }}$ was of $3 \mathrm{MAC}$ in the first minute. Then, the flow was reduced to $1 \mathrm{~L} \cdot \mathrm{min}^{-1}$ until the $20^{\text {th }}$ minute and $F_{\text {adm }}$ was decreased to $1.5 \mathrm{MAC}$ until the $10^{\text {th }}$ minute and to 1.2 MAC until the end.

Patients were maintained in mechanical ventilation with respiratory rate (RR) of 10 cycles per minute (c.p.m.) and tidal volume $(\mathrm{Vt})$ of $10 \mathrm{ml} \cdot \mathrm{kg}^{-1}\left(100 \mathrm{ml} \cdot \mathrm{kg}^{-1} \cdot \mathrm{min}^{-1}\right)$.

Data were collected during the first 20 minutes of anesthesia and liquid anesthetic volume consumption was measured for all anesthetic agents, except for desflurane due to technical problems (extremely volatile anesthetic, vaporizer and bottle with valves). For desflurane, the volume was calculated by the formula:

$$
V=\frac{\text { Concentration flow time }}{\frac{D 22.4(273 t)}{M W 273}}
$$

(Concentration in \%/100; flow in $\mathrm{ml} / \mathrm{min}$; time in minutes; $\mathrm{V}=$ volume $\mathrm{D}=$ density; $\mathrm{MW}=$ molecular weight; and $\mathrm{t}=$ temperature in $\left.{ }^{\circ} \mathrm{C}\right)^{4}$.

Statistical analysis was performed with analysis of variance using $\mathrm{F}$ test and considering significant $\alpha=0.05(5 \%)$.

\section{RESULTS}

There were no statistical differences among groups in weight and age (Table II). Anesthetic consumption was obtained by volume measurements in 20 minutes (Table III) and can be considered low compared to the literature reports ${ }^{7}$.

Table III - Anesthetic Volume Consumption (ml) in 20 Minutes $($ Mean \pm SD)

\begin{tabular}{lc}
\hline Groups & Volume Consumption \\
\hline Halothane & $816 \pm 3.42$ \\
Isoflurane & $11.31 \pm 5.08$ \\
Sevoflurane & $9.63 \pm 5.81$ \\
Desflurane * & $11.89 \pm 0.65$ \\
\hline
\end{tabular}

${ }^{*}$ Calculated volume

Table II - Age and Weight (Mean \pm SD)

\begin{tabular}{lcccc}
\hline Groups & Halothane & Isoflurane & Sevoflurane & Desflurane \\
\hline Age (years) & $31.14 \pm 11.91$ & $29.57 \pm 8.64$ & $30.86 \pm 12.86$ & $29.14 \pm 12.98$ \\
Weight $(\mathrm{kg})$ & $62.14 \pm 17.32$ & $65.71 \pm 9.18$ & $62 \pm 7.64$ & $65.42 \pm 15.74$ \\
\hline
\end{tabular}


Regarding hemodynamic variables, mean blood pressure (Figure 2) varied from 98 to $63 \mathrm{mmHg}$. There was one case of a $22.56 \%$ pressure decrease after 9 minutes in the halothane group (BP $69 / 35 \mathrm{mmHg}$ ) which required $10 \mathrm{mg}$ ephedrine, followed by an improvement (BP $83 / 41 \mathrm{mmHg}$ ). Heart rate (Figure 3) varied from 98 to $68 \mathrm{bpm}$. In 1 sevoflurane group patient there was a $25.39 \%$ decrease after 17 minutes but without clinical relevance. Regarding ventilatory variables, end expired $\mathrm{CO}_{2}$ fractional (Figure 4) varied from 35 to 30 $\mathrm{mmHg}$, also without clinical relevance.

In the halothane group, FA/MAC ratio equal to 1 , that is, 1 MAC, was reached in 2 minutes, varying from 1.21 to 1.47 MAC during the first 20 minutes, which may be considered normal for a clinically stable anesthesia, considering that Anesthetic Dose ${ }_{95}\left(A_{95}\right)$ is approximately $1.3 \mathrm{MAC}^{8}$ (Figure 5 ). In the isoflurane group, FA/MAC ratio equal to 1 was reached in 1 minute and varied from 1.11 to $1.21 \mathrm{MAC}$ during the 20 minutes (Figure 6).

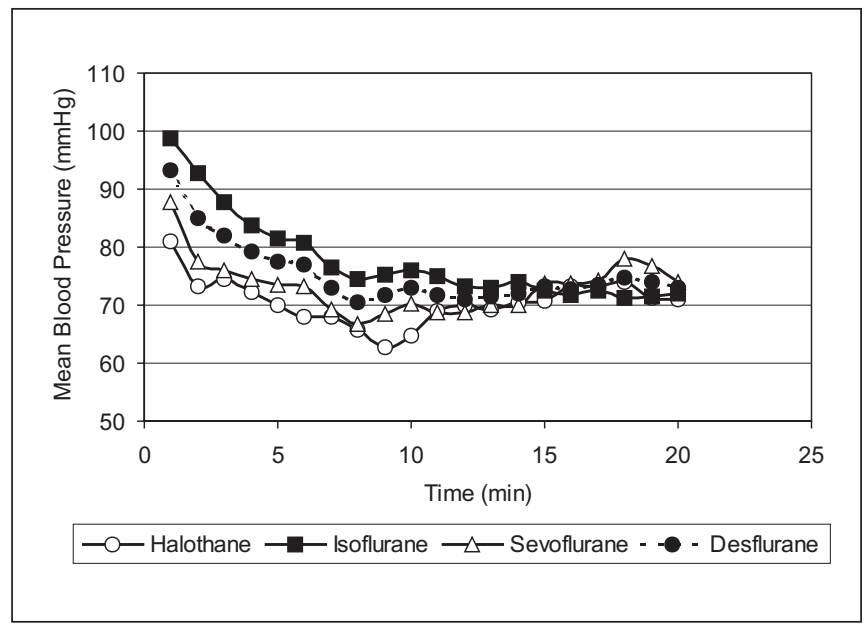

Figure 2 - Mean Blood Pressure During First 20 Minutes

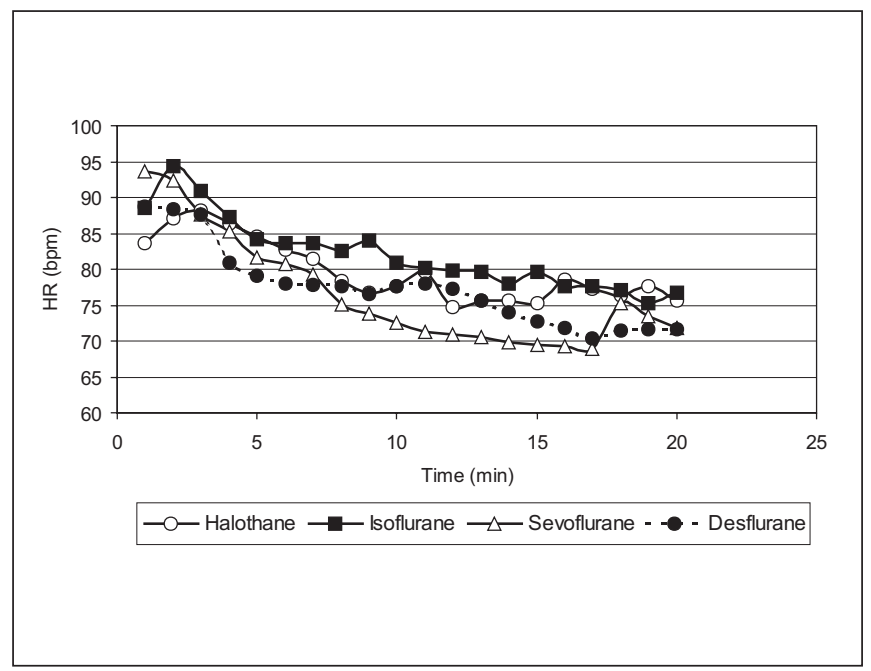

Figure 3 - Heart Rate During First 20 Minutes

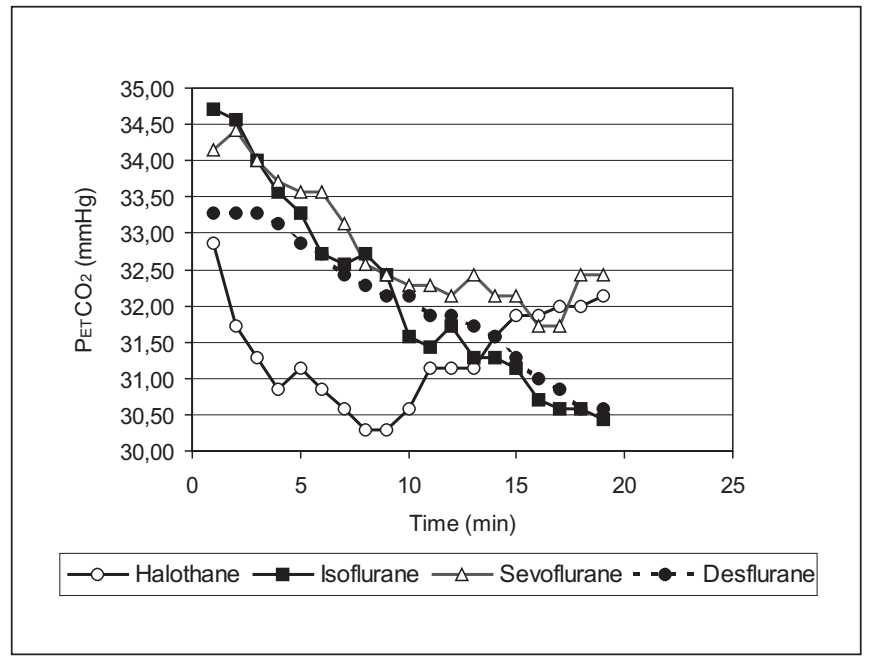

Figure $4-\mathrm{CO}_{2}$ Expired Fraction During First 20 Minutes

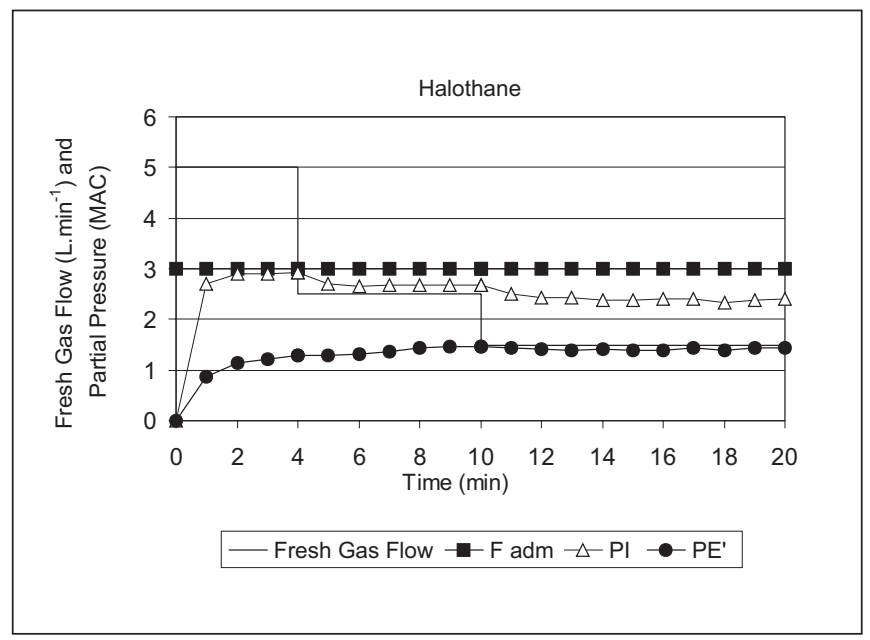

Figure 5 - Results Obtained with Halothane

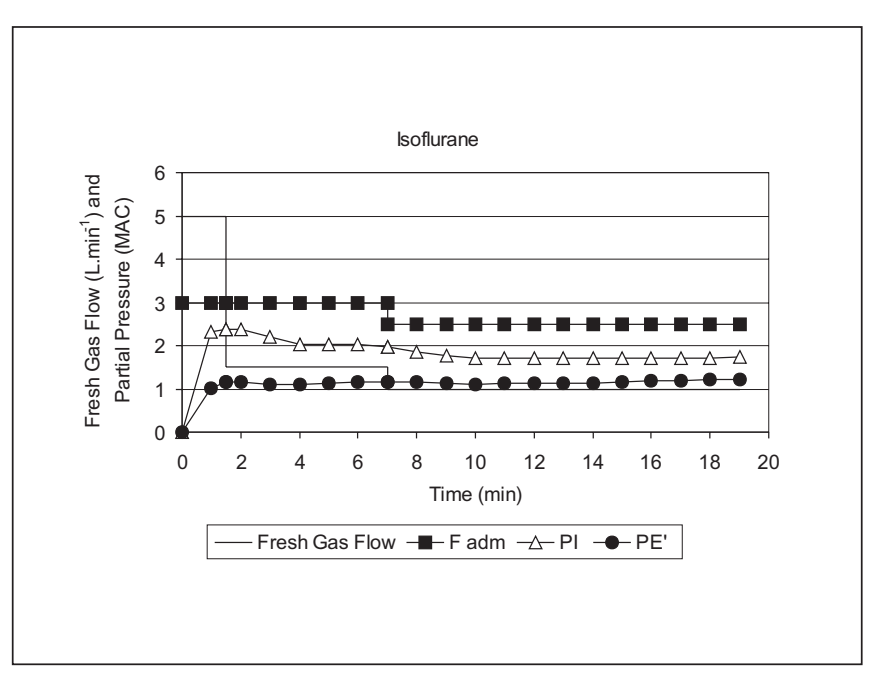

Figure 6 - Results Obtained with Isoflurane 
In the sevoflurane group, FA/MAC ratio was 1.53 in one minute and FAvaried from 1.10 to 1.34 MAC for 20 minutes (Figure 7). In the desflurane group, FA/MAC ratio was equal to 0.94 in 1 minute and FA varied from 1.07 to 1.14 MAC (Figure 8) for 20 minutes.

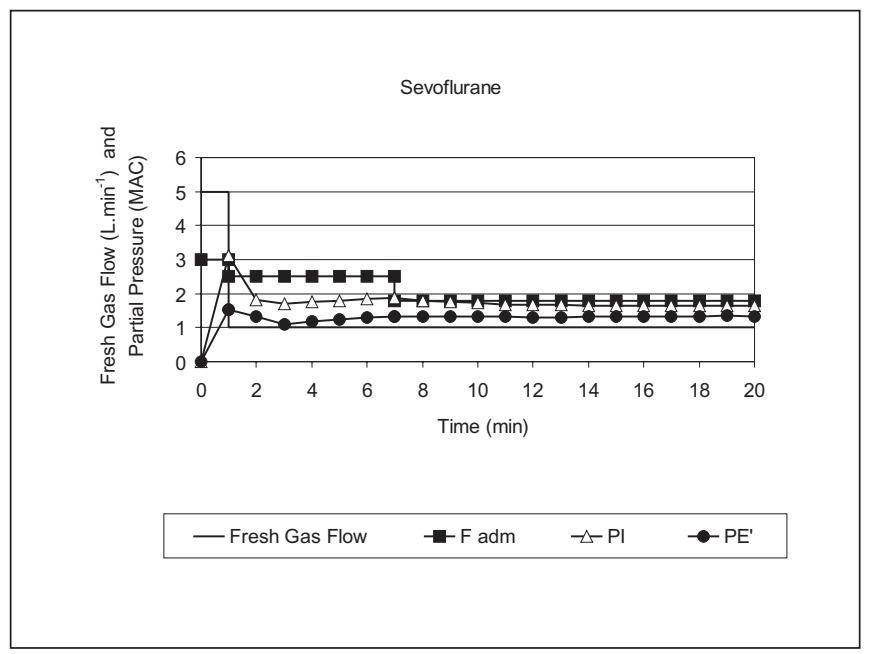

Figure 7 - Results Obtained with Sevoflurane

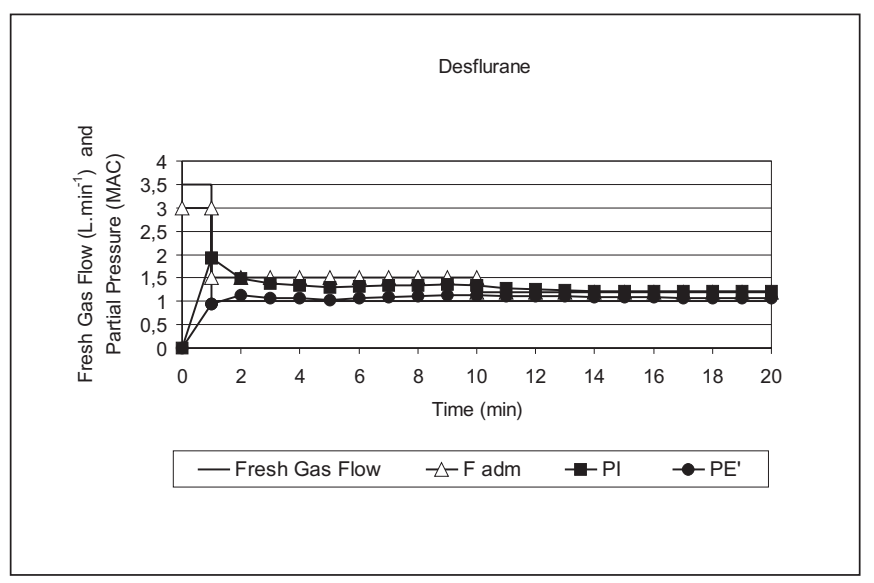

Figura 8 - Results Obtained With Desflurane

\section{DISCUSSION}

Using Mapleson's theoretical study, fresh gas flow and anesthetic concentrations (fractional) were evaluated in an attempt to improve their use during the first minutes of anesthesia in order to obtain a fast induction with a minimum volatile anesthetics consumption ${ }^{5}$. This rapid induction requires high fresh gas flow (FGF) and high anesthetics fractional $\left(F_{a d m}\right)$ to obtain a rapid kinetic balance between this fractional and the inspired fractional $\left(F_{I}\right)$ and then, through efficient alveolar ventilation, to bring alveolar fractional (FA) closer to $\mathrm{F}_{1}$.
This theoretical model is able to simulate volatile anesthetics uptake (absorption) and distribution through data obtained from physiological variables: ventilation, dead space, cardiac output and organs blood perfusion, based on a standard 40 years old man weighing $70 \mathrm{~kg}^{9}$. Values of anesthesia machine components volume and of agents solubility, expressed by blood/gas and tissue/blood partition coefficient of each organ, were obtained from the literature ${ }^{10,11}$.

In the model, the respiratory system has three compartments, as follows: the anesthesia machine (including mechanical dead space), anatomic dead space and pulmonary alveolus air. Circulation and tissues have multiple compartments and are laid in series, according to blood perfusion: brain, viscera, muscles and fat tissue. They are connected to arterial blood perfusion which brings the anesthetic drug from the alveolus to the organs, and to venous system, which takes the anesthetic drug back to the alveolus.

Simulation is performed by imagining the anesthetic drug being taken as a vapor from the vaporizer to the respiratory system by the fresh gas flow, and from this to the pulmonary alveolus through alveolar ventilation, being then removed by the blood and distributed to the organs according to blood perfusion and solubility. During this trip, the anesthetic drug suffers the effect of dilution in the anesthesia machine and the alveolus. Alveolar ventilation is the major factor determining the kinetic balance between alveolar and inspired fractional.

The alveolar anesthetic uptake by the blood to distribute it throughout the body is accounted for in this model. Since brain perfusion is very high, the brain-alveolar balance is promptly reached. Hence, alveolar anesthetic concentration, that is, end expired fractional, expresses brain fractional.

When starting inhalational anesthetics administration, there is a great difference between administered fractional, which immediately leaves the vaporizer, and the inspired fractional, at patient's mouth level after going through the inhalational system of the anesthesia machine, as well as between it and alveolar fractional which of course, is still for below MAC of the agent being used. These differences are explained by the lack of anesthetics in the anesthesia machine inhalation system and in the alveolar air at this point in time.

$F_{A}$ equal or close to MAC is the first goal to be met during inhalational anesthesia. For such, a very high anesthetic offering is needed for the inhalation system through fresh gas flow and from it to the pulmonary alveolus through alveolar ventilation.

Considering this pharmacokinetic concept, Mapleson's theoretical model accounts for obtaining 1 MAC during the first minutes, depending on agents' solubility. This is why fresh gas flow is high, equal to pulmonary minute ventilation, and $F_{a d m}$ is equal to $3 \mathrm{MAC}$. The idea was also to keep alveolar ventilation within normal standards to maintain patients in normocapnia or mild hypocapnia and take to the alveolus the volume of anesthetics needed for MAC to be promptly reached. 
Using FGF and $F_{\text {adm }}$ values in the moments scheduled by the method and described on the results, FA/MAC ratios were obtained and their peaks varied from 0.94 to 1.5 (mean of 1.2). These results were very close to Mapleson's study, in which the target was an FA/MAC ratio of 1 to be obtained during the first minutes and then maintained constant.

Halothane, a more soluble agent, reached MAC in 2 minutes. The other anesthetics (isoflurane, sevoflurane and desflurane) reached MAC in 1 minute. Since they are less soluble agents, the balance between alveolar partial pressure and inspired partial pressure is faster.

The difference between our results and those of Mapleson's theoretical study was certainly due to the fact that our sample was not standardized (40 years old and $70 \mathrm{~kg}$ men). Patients were ordinary people found in our daily practice. Even so, it must be stressed that differences between results were minor and without statistical significance.

Drug consumption during the first minutes of anesthesia was high due to the high fresh gas flow. When the flow was decreased, obviously consumption also decreased. Regardless of this higher volume used in the beginning of anesthesia, mean anesthetic volume consumption during 20 minutes was low.

It is important to stress that, to effectively and safely use this method, it is advisable to have available an anesthetic gas monitor to continuously measure end expired fractionals of such agents, in addition to a complete anesthesia machine with standard monitoring, such as pulse oximetry and capnography. Currently, this equipment should be part of the anesthesiologist routine whenever possible.

Our results have confirmed the clinical feasibility of Mapleson's theoretical model. A fast increase in inhalational agent end expired fractional (alveolar) was obtained, which reached $1 \mathrm{MAC}$ already during the first minute, being then maintained constant. Anesthetic consumption may be considered among the lowest ever described.

\section{REFERÊNCIAS - REFERENCES}

01. Snow J - On Chloroform and the Other Anesthetics. London, J.Churchill, 1858;58-74.

02. Waters RM - Clinical scope and utility of carbon dioxide filtration in inhalation anesthesia. Curr Res Analg Anesth ,1923;3:20-26.

03. Lucas GHW - The discovery of ciclopropane. Curr Res Anesth Analg, 1961;40:15-22.

04. Lowe HJ, Ernst EA - The Quantitative Practice of Anesthesia, Use of Closed Circuit, Baltimore, Maryland, The William and Wilkins Co, 1981;1-26.

05. Mapleson WW - The theoretical ideal fresh-gas flow sequence at the start of low-flow anaesthesia. Anaesthesia, 1998;53: 264-272.

06. Mapleson WW - Effect of age on MAC humans :a meta analysis. Br J Anaesth, 1996;76:179-185.

07. Silva JMC, Pereira E, Saraiva RA - Consumo de anestésicos inalatórios no Brasil. Rev Bras Anestesiol, 1982;32:431- 440.

08 de Jong RH, Eger E I,II - MAC expandend AD 50 and AD 95 values of common inhalation anesthetics in man. Anesthesiology, 1975;42:384-389.
09. Davis NR, Mapleson WW - Structure and quantification of a physiological model of the distribution of injected agents and inhaled anaesthetics. Br J Anaesth, 1981;53:399-405.

10. Mapleson WW - An electric analogue for uptake and exchange of inert gases and other agents. J Appl Physiol, 1963;18: 197-204.

11. Lerman J, Schmitt-Bantel BI, Gregory GA et al - Effect of age on the solubility of volatile anesthetics in human tissues. Anesthesiology ,1986;65:307-311.

\section{RESUMEN}

Borges MMJ, Saraiva RA - Secuencia de Flujo de Gas Fresco para Inicio de la Anestesia con Bajo Flujo: Aplicación Clínica del Estudio Teórico de Mapleson

Justificativa y Objetivos - En estudio teórico, Mapleson utilizando un modelo farmacocinético multicompartimental, con un hombre padrón de 40 años y $70 \mathrm{~kg}$, demostró que, con flujo de gas fresco (FGF) inicial igual a la ventilación pulmonar total, siendo después reducido hasta 1 L. $\min ^{-1}$ y concentración (Fraccional) administrada del anestésico $\left(F_{\text {adm }}\right)$ igual a 3 CAM, la fraccional expirada final, también expresa como alveolar $\left(F_{E}{ }^{\prime}=F_{A}\right)$, puede llegar a 1 CAM en pocos minutos, de acuerdo con la solubilidad del agente inhalado. El objetivo del presente trabajo fue realizar la aplicación clínica de este modelo teórico.

Método - Después de la aprobación por la comisión de Ética, fueron estudiados 28 pacientes de ambos sexos, con edad entre 18 y 55 años, sometidos a anestesia general, divididos aleatoriamente en 4 grupos de 7 pacientes de acuerdo con anestésico utilizado (halotano, isoflurano, sevoflurano y desflurano). La inducción fue venosa con propofol, fentanil y vecuronio. Y manutención con el agente inhalatorio diluido en oxígeno, bajo ventilación pulmonar mecánica. Los parámetros fueron los siguientes, de acuerdo con el agente utilizado: Grupo del halotano: FGF inicial de 5 L. min $^{-1}$ hasta 4 minutos, seguido por 2,5 L. min ${ }^{-1}$ hasta 10 minutos y 1,5 L. min ${ }^{-1}$ hasta 20 minutos, $F_{\text {adm }}$ igual a 3 CAM durante los 20 minutos iniciales de la anestesia. Grupo del isoflurano: el FGF inicial fue de 5 L. min ${ }^{-1}$ por 1,5 minuto, seguido por 1,5 L. min ${ }^{-1}$ hasta 7 minutos y 1 L. min ${ }^{-1}$ hasta 20 minutos. La $F_{a d m}$ fue de 3 CAM hasta 7 minutos y 2,5 CAM hasta el vigésimo minuto. Grupo del sevoflurano: el FGF inicial fue de $5{\mathrm{~L} . \mathrm{min}^{-1}}^{\text {por }} 1$ minuto y 1 L. min $^{-1}$ hasta el vigésimo minuto y la $F F_{\text {adm }}$ de 3 CAM por 1 minuto, después 2,5 CAM hasta 7 minutos y 1,8 CAM hasta 20 minutos. Grupo del desflurano: el FGF inicial fue de 3,5 L. min $^{-1}$ por 1 minuto y 1 L. $\mathrm{min}^{-1}$ hasta completar los 20 minutos y la $F_{a d m}$ de 3 CAM por 1 minuto, seguido de 1,5 CAM hasta 10 minutos y 1,2 CAM hasta 20 minutos. Además de la monitorización rutinera de las variables fisiológicas (cardiovasculares e respiratorias) fueron medidas FI, y FE' (FA) de los agentes utilizados.

Resultados - Grupo del halotano: la FA llegó a 1,15 CAM en 2 minutos y varió de 1,21 a 1,47 CAM hasta 20 minutos. Grupo del isoflurano: la FA fue de 1,03 CAM en 1 minuto, variando de 1,11 a 1,21 CAM hasta 20 minutos. Grupo del sevoflurano: Ia FA de 1,53 CAM fue alcanzada en 1 minuto, variando de 1,10 a 1.34 CAM durante los 19 minutos restantes. Grupo del desflurano: la FA fue de 0.94 CAM en 1 minuto, variando de 1,07 hasta 1,14 CAM hasta el vigésimo minuto.

Conclusiones - Los resultados obtenidos comprueban la aplicabilidad clínica del modelo teórico de Mapleson. De esta manera, se consiguió un rápido aumento de la FA del agente inhalatorio que llegó a 1 CAM, en 1 a 2 minutos, manteniéndose en este valor con pequeñas oscilaciones y bajo consumo de anestésico. 